\title{
Use of Cyclic Backbone NGR-Based SPECT to Increase Efficacy of Postmyocardial Infarction Angiogenesis Imaging
}

\author{
Geert Hendrikx, ${ }^{1}$ Tilman M. Hackeng, ${ }^{2}$ Rick van Gorp, ${ }^{2}$ \\ Matthias Bauwens, ${ }^{1}$ Leon J. Schurgers, ${ }^{2}$ Felix M. Mottaghy, ${ }^{1,3}$ \\ Mark J. Post, ${ }^{4}$ and Ingrid Dijkgraaf ${ }^{2}$ \\ ${ }^{1}$ Department of Radiology and Nuclear Medicine, Cardiovascular Research Institute Maastricht (CARIM), \\ Maastricht University Medical Center (MUMC+), Maastricht, Netherlands \\ ${ }^{2}$ Department of Biochemistry, Cardiovascular Research Institute Maastricht (CARIM), \\ Maastricht University Medical Center (MUMC+), Maastricht, Netherlands \\ ${ }^{3}$ Department of Nuclear Medicine, University Hospital, RWTH University, Aachen, Germany \\ ${ }^{4}$ Department of Physiology, Cardiovascular Research Institute Maastricht (CARIM), \\ Maastricht University Medical Center (MUMC+), Maastricht, Netherlands \\ Correspondence should be addressed to Ingrid Dijkgraaf; i.dijkgraaf@maastrichtuniversity.nl
}

Received 29 May 2017; Revised 9 September 2017; Accepted 19 September 2017; Published 24 October 2017

Academic Editor: Barbara Palumbo

Copyright (c) 2017 Geert Hendrikx et al. This is an open access article distributed under the Creative Commons Attribution License, which permits unrestricted use, distribution, and reproduction in any medium, provided the original work is properly cited.

As CD13 is selectively expressed in angiogenesis, it can serve as a target for molecular imaging tracers to noninvasively visualize angiogenic processes in vivo. The CD13-targeting moiety NGR was synthesized and cyclized by native chemical ligation (NCL) instead of disulfide bridging, leading to a cyclic peptide backbone: cyclo(Cys-Asn-Gly-Arg-Gly) (coNGR). Beside this new monomeric coNGR, a tetrameric NGR peptide co(NGR) ${ }_{4}$ was designed and synthesized. After radiolabeling, their in vitro and in vivo characteristics were determined. Both coNGR-based imaging agents displayed considerably higher standardized uptake values (SUVs) at infarcted areas compared to the previously reported disulfide-cyclized cNGR imaging agent. Uptake patterns of ${ }^{111}$ In-coNGR and ${ }^{111} \mathrm{In}-\mathrm{co}(\mathrm{NGR})_{4}$ coincided with CD13 immunohistochemistry on excised hearts. Blood stability tests indicated better stability for both novel imaging agents after $50 \mathrm{~min}$ blood incubation compared to the disulfide-cyclized cNGR imaging agent. In mice, both coNGR peptides cleared rapidly from the blood mainly via the kidneys. In addition, co(NGR) ${ }_{4}$ showed a significantly higher specific uptake in infarcted myocardium compared to coNGR and thus is a promising sensitive imaging agent for detection of angiogenesis in infarcted myocardium.

\section{Introduction}

Angiogenesis is an endogenous healing process which serves to restore tissue blood supply in response to ischemic injury [1]. The extent of angiogenic activity is correlated with infarct healing and postmyocardial infarction (MI) remodeling [2]. Augmentation of experimental angiogenesis has several beneficial effects on post-MI remodeling, including reduced apoptosis of hypertrophic cardiomyocytes in the border zone, attenuated collagen deposition and scar formation in the noninfarcted zone, and improved long-term ventricular function $[2,3]$. However, while positive results were shown in animal models of MI, the benefit for MI patients has yet to be shown. So far, results from double-blind placebocontroled trials were disappointing [4-7]. These negative outcomes were most likely attributable to a combination of factors, including patient selection, choice of delivery platforms for therapeutic agents, and, importantly, lack of sensitive noninvasive detection methods for angiogenesis [8].

Frequently employed imaging agents for noninvasive nuclear imaging of cardiovascular angiogenesis in animal models are based either on the $\alpha_{\mathrm{v}} \beta_{3}$ integrin-targeting 
<smiles>CC(C)CC(NC(=O)CCSCCNC(=O)C(CCCNC(=N)N)NC(=O)CNC(=O)C(CC(N)=O)NC(=O)C(N)CSc1ccccc1)C(N)=O</smiles>

FIgURE 1: Reaction mechanism of cyclization of CNGRG-MpaL via NCL.

Arg-Gly-Asp (RGD) amino acid sequence or on vascular endothelial growth factor (VEGF) [9]. Several single-photon emission computed tomography (SPECT) and positron emission tomography (PET) based studies reported positive results with RGD targeting agents (reviewed in $[10,11]$ ). However, in competition studies, the RGD motif was found to have a lower target homing ratio (target to control tissue) compared to the Asn-Gly-Arg (NGR) tripeptide sequence which binds to selectively upregulated CD13 on angiogenically active endothelial cells [12]. Given the high target homing ratio of cyclic NGR peptides, employing a cyclic NGRbased imaging agent might result in better image quality.

The CD13-targeting NGR motif has been explored at our institute as a molecular angiogenesis imaging agent for fluorescence microscopy [13], magnetic resonance imaging (MRI) [14], and more recently single-photon emission computed tomography (SPECT) imaging [15]. These cyclic NGRbased molecular imaging agents showed enhanced uptake in the infarct area and infarct border zone 7 days after MI in a mouse model [13-15]. Although each study established specific binding of their respective NGR-based ligand in the infarct area and infarct border zone, none of these molecular imaging agents had the potential for clinical translation at this stage. While localizing the fluorescently labeled NGR-based molecular imaging probe would require an invasive procedure, the cadmium-selenium core of cyclic NGR-conjugated paramagnetic quantum dots ( $\mathrm{pQD}$ ) in combination with their accumulation in liver and spleen hampers translation of the MRI angiogenesis imaging agent into clinical trials. For the NGR-based SPECT tracer to be considered as a potential clinical angiogenesis imaging tool, specific target uptake has to increase.

Whereas the ring structure of our previously developed cyclic NGR peptide cyclic(NAc-Cys-Asn-Gly-Arg-Cys-GlyGly-Lys) was cyclized using a disulfide bond (henceforth called "cNGR"), we now cyclized the ring structure via a peptide bond by native chemical ligation (NCL, Figure 1) resulting in a new monomeric tracer: cyclo(CNGRG) ("coNGR"). Theoretically, this would lead to a more stable molecule and higher target uptake in vivo. Because a multimerization strategy has been shown to be beneficial for targeting and imaging with RGD peptides [16, 17], coNGR peptide moieties were coupled to a lysine wedge, resulting in a tetrameric coNGR imaging agent: cyclo(CNGRG) ${ }_{4}$ ("tetrameric coNGR" or "co(NGR),"). Both monomeric coNGR and tetrameric coNGR were conjugated with diethylene triamine pentaacetic acid (DTPA, Figures 2(a) and 2(b)), radiolabeled with ${ }^{111} \mathrm{In}$, and in vitro and in vivo studies were performed. Using dualisotope SPECT imaging, these new angiogenesis imaging agents were tested in a mouse model for MI in combination with the perfusion tracer ${ }^{99 \mathrm{~m}} \mathrm{Tc}$-sestamibi.

\section{Materials and Methods}

\subsection{Synthesis of coNGR and $\operatorname{co}(N G R)_{4}$}

2.1.1. Synthesis of Linear H-[Cys $\left.{ }^{1}-\mathrm{Asn}^{2}-G l y^{3}-\mathrm{Arg}^{4}-G l y^{5}\right]$ $\mathrm{MpaL}-\mathrm{NH}_{2}$. Boc-Cys(MeBzl)-OH, Boc-Asn(Xan)-OH, and Boc-Arg(Tos)-OH were purchased from Bachem (Bubendorf, Switzerland). Boc-Gly-OH was obtained from Peptide Institute, Inc. (Osaka, Japan).

Linear CNGRG-MpaL thioester peptide was synthesized on methylbenzhydrylamine- (MBHA-) polystyrene resin (ChemPep, Wellinton, FL, USA; $0.2-0.4 \mathrm{mmol}$ scale) as described previously $[18,19]$. Instead of 2 -( $1 H$-benzotriazole-1-yl)-1,1,3,3-tetramethyluronium hexafluorophosphate (HBTU), 2-(6-Chloro-1H-benzotriazol-1-yl)-1,1,3,3-tetramethylaminium hexafluorophosphate (HCTU, Peptides International, Louisville, KY, USA) was used as coupling reagent. After the peptide was cleaved from the resin by treatment with anhydrous HF (GHC Gerling, Holz \& Co. Handels $\mathrm{GmbH}$, Hamburg, Germany), the crude product was analyzed on a Waters ${ }^{\mathrm{TM}}$ (Milford, MA, USA, and Etten-Leur, The Netherlands) ultrahigh performance liquid chromatography mass spectrometry (UPLC-MS) XEVOG2QToF system. The peptide was purified by semipreparative HPLC using Vydac $\mathrm{C}_{18}$ HPLC columns $(10 \mathrm{~mm} \times 250 \mathrm{~mm}$, $12 \mathrm{~mL} / \mathrm{min}$ flow rate or $22 \mathrm{~mm} \times 250 \mathrm{~mm}, 20 \mathrm{~mL} / \mathrm{min}$ flow rate; Grace Davison Discovery Sciences, Deerfield, IL, USA) connected to a Waters Deltaprep System consisting of a Waters Prep LC Controller and a Waters 2487 Dual wavelength Absorbance Detector $(\lambda=214 \mathrm{~nm})$. To elute the peptide, an appropriate gradient of buffer B in buffer A, where buffer $\mathrm{A}$ is $0.1 \%$ trifluoroacetic acid (TFA, Biosolve BV, Valkenswaard, The Netherlands) in $\mathrm{H}_{2} \mathrm{O} / \mathrm{CH}_{3} \mathrm{CN}(95 / 5$, v/v, 


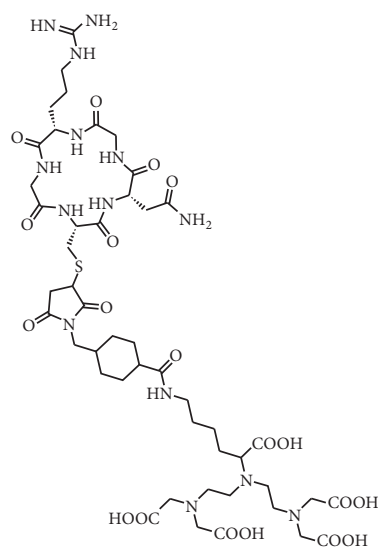

(a)

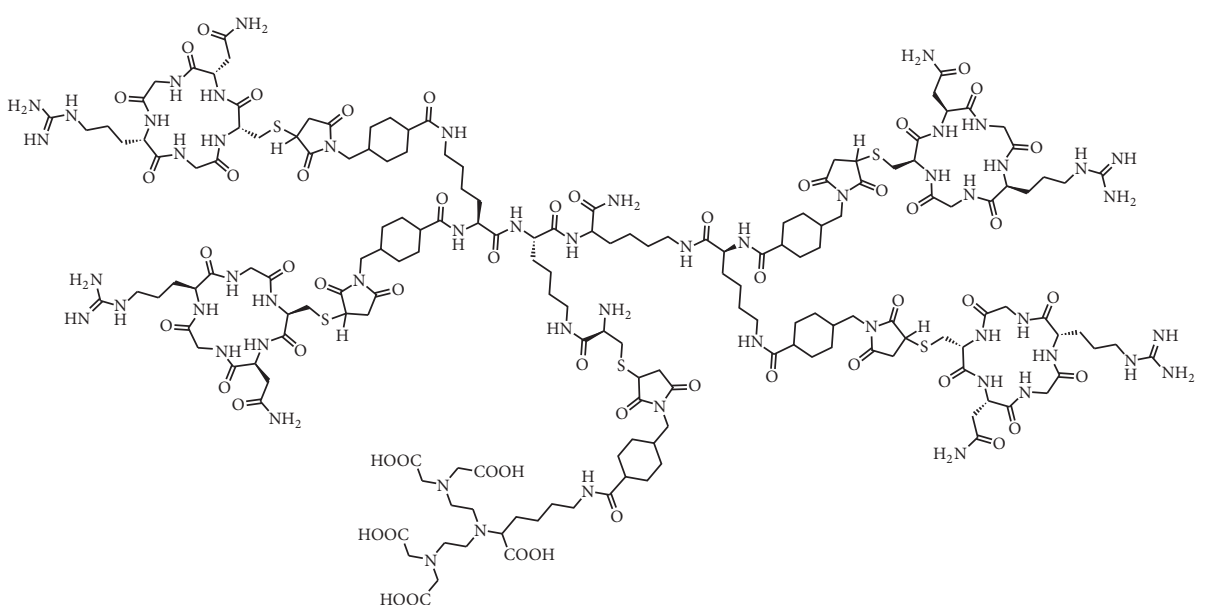

(b)

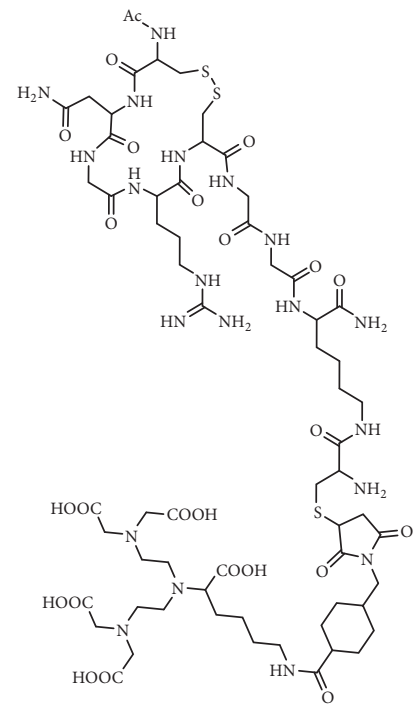

(c)

Figure 2: Structural formulas of DTPA-conjugated coNGR (a), co(NGR) ${ }_{4}$ (b), and cNGR (c).

Biosolve) and buffer $\mathrm{B}$ is $0.1 \%$ TFA in $\mathrm{CH}_{3} \mathrm{CN} / \mathrm{H}_{2} \mathrm{O}(90 / 10$, v/v), was used.

2.1.2. Cyclization of $H-[C-N-G-R-G]-M p a L-N H_{2}$ by NCL. For cyclization, the peptide was dissolved in $50 \mathrm{mM}\left(\mathrm{NH}_{4}\right)_{2} \mathrm{CO}_{3}$ pH 7.8 (Sigma Aldrich, Steinheim, Germany) at a maximum concentration of $1 \mathrm{mg} / \mathrm{mL}$. The reaction was performed at $37^{\circ} \mathrm{C}$ and was followed over time by UPLC-MS analysis. Generally, cyclization was complete after $1 \mathrm{~h}$. After cyclization, coNGR was purified as described above.

2.1.3. $\operatorname{co}(\mathrm{NGR})_{4}$. To synthesize a tetrameric cyclic NGR peptide, a scaffold peptide with 4 NGR-coupling sites was necessary. Therefore, a lysine wedge was synthesized on MBHA resin (0.2 mmol scale). First, Boc-Lys(Fmoc)-OH (Bachem; Fmoc =9-fluorenylmethoxycarbonyl) was coupled to the solid support, followed by two coupling cycles of Boc-Lys(Boc)-OH (Bachem). After chain assembly of the
$N^{\alpha}$-Boc protected lysine wedge, $313 \mathrm{mg}$ peptidyl-resin was treated with piperidine $20 \mathrm{v}-\%$ in DMF $(4 \times 3 \mathrm{~min}$, both Biosolve) for $N^{\varepsilon}$-Fmoc group removal. Then, Boc-thiazolidine4-carboxylic acid (Thz; Bachem; $259 \mathrm{mg}$ ) was coupled using $2 \mathrm{~mL} 0.5 \mathrm{M}$ HCTU and $400 \mu \mathrm{l} N, N$-Diisopropylethylamine (DiPEA; Biosolve). After treatment of the peptidyl-resin with TFA $(2 \times 1 \mathrm{~min})$ for $N^{\alpha}$-Boc-deprotection of the 4 lysine residues and coupling of succinimidyl $4-(N-$ maleimidomethyl)cyclohexane-1-carboxylate (SMCC; $1.47 \mathrm{~g}$ in $6 \mathrm{~mL} \mathrm{DMF}$ ), the peptidyl-resin was cleaved from the resin using HF. Subsequently, the crude Lys(Thz)-(Lys) $)_{2}$-(SMCC) product was purified on semipreparative RP-HPLC. For synthesis of the tetrameric coNGR peptide, $5.3 \mathrm{mg}$ coNGR and $1.7 \mathrm{mg}$ of functionalized lysine wedge were dissolved in $1 \mathrm{~mL} 0.1 \mathrm{M}$ sodium phosphate buffer $\mathrm{pH} 6.5$, containing $6 \mathrm{M}$ $\mathrm{GuHCl}$ (Sigma Aldrich). After $3 \mathrm{~h}$ at $37^{\circ} \mathrm{C}$, product formation was confirmed on UPLC-MS and the reaction mixture was purified on semipreparative HPLC as described above. 
TABle 1: Analytical mass data of coNGR-based tracer constructs. Masses are given in Da. From top to bottom, mass of linear peptide (thioester), cyclic peptide after NCL, and the lysine wedge with four SMCC linkers are represented. Last two rows represent mass of DTPAcoNGR and DTPA-co(NGR) ${ }_{4}$, respectively.

\begin{tabular}{lccc}
\hline Compound & Monoisotopic mass & Molecular weight & Measured \\
\hline CNGRG-MpaL & 705.31 & 705.81 & 705.32 \\
coNGR & 487.20 & 487.54 & 487.22 \\
Lys(Thz)-(Lys) ${ }_{2}$-(SMCC) $)_{4}$ & 1520.77 & 1521.84 & 1520.83 \\
DTPA-coNGR & 1170.50 & 1171.25 & 1170.49 \\
DTPA-co(NGR) & 4140.86 & 4143.69 & 4140.76 \\
\hline
\end{tabular}

2.1.4. Maleimide-DTPA Coupling. Maleimide-DTPA was prepared via the method described by Dirksen et al. [20, 21]. coNGR or $\operatorname{co}(\mathrm{NGR})_{4}$ and maleimide-DTPA (1.5 eq) were dissolved in $0.1 \mathrm{M}$ acetate $\mathrm{pH} 4, \mathrm{MeONH}_{2}$, EDTA, $6 \mathrm{M}$ $\mathrm{GuHCl}$. Both reactions were performed at $37^{\circ} \mathrm{C}$ and followed on UPLC-MS. After reaction completion, the reaction mixture was purified on semipreparative HPLC.

Analytical mass data of linear CNGRG-MpaL, coNGR, Lys(Thz)-(Lys) $)_{2}-(\mathrm{SMCC})_{4}$, DTPA-coNGR, and DTPA$\mathrm{co}(\mathrm{NGR})_{4}$ are given in Table 1.

\subsection{Imaging Probes}

2.2.1. ${ }^{111} I n-c o N G R$ and ${ }^{111} I n-c o(N G R)_{4}$. coNGR and co(NGR) $)_{4}$ were radiolabeled with ${ }^{111} \mathrm{InCl}_{3}$ (Mallinckrodt, Petten, The Netherlands) analogous to what has been described previously [15]. cNGR was used as reference compound and labeled with ${ }^{111} \mathrm{InCl}_{3}$ as described previously [15].

2.2.2. Stability Tests. ${ }^{111}$ In-cNGR, ${ }^{111}$ In-coNGR, or ${ }^{111}$ Inco(NGR) $)_{4}$ was added to $1 \times 10 \mathrm{~mL}$ human blood in heparin (BD Biosciences, Vianen, The Netherlands). Blood stability was tested at $0,10,30$, and $50 \mathrm{~min}$. For each time point imaging agents were separated from blood cells and proteins by adding $0.5 \mathrm{~mL} \mathrm{MeCN}$ (VWR International BV, Breda, The Netherlands) to $0.5 \mathrm{~mL}$ blood sample followed by centrifugation $(2,000 \times \mathrm{g}, 3 \mathrm{~min})$. Supernatant samples were analyzed by HPLC using the above-mentioned method.

2.2.3. Octanol/Water Partition Coefficient. The $\log P$ value of each compound was determined in three separate experiments as described previously [22].

2.2.4. ${ }^{99 m}$ Tc-Sestamibi. Freshly prepared ${ }^{99 m}$ Tc-sestamibi (Technescan sestamibi) was ordered from GE Healthcare (Eindhoven, The Netherlands).

2.3. Animal Studies. In 10-12-week-old male Swiss mice, we induced MI by ligation of the left anterior descending coronary artery (LAD) as described before [23] to test coNGR $(n=5)$ or $\operatorname{co}(\mathrm{NGR})_{4}(n=8)$. A group of sham-operated Swiss mice $(n=5)$ was used as control. SPECT imaging was performed 7 days after MI or sham surgery. An overview of the number of animals used per tracer is given in Table S1. All animals were held under the guidelines of the animal care facility (Maastricht University). All animal experiments were approved by the Committee for Animal Welfare of Maastricht University.

2.4. Micro-SPECT. Mice were anesthetized with isoflurane (induction 2.5\%; maintenance 1.5\%), a catheter was placed in a tail vein, and animals were positioned in the SPECT camera (MILabs, Utrecht, The Netherlands). Prior to image acquisition, a bolus injection of ${ }^{99 \mathrm{~m}}$ Tc-sestamibi and ${ }^{111}$ In-coNGR or ${ }^{111}$ In-co(NGR) $)_{4}$ was given intravenously (i.v.) in a maximum volume of $200 \mu \mathrm{L}$. Table S1 in Supplementary Material available online at https://doi.org/10.1155/2017/8638549 provides an overview of the average injected dose. Immediately after injection, 4 consecutive time frames of $15 \mathrm{~min}$ each were acquired. For image quantification we used the last of these frames.

2.5. SPECT Image Reconstruction. Acquired list mode data was reconstructed using MILabs reconstruction software (version 2.51) employing the POS-EM algorithm (6 iterations and 16 subsets, reconstructed at a voxel size of $0.4 \mathrm{~mm}) .{ }^{99 \mathrm{~m}} \mathrm{Tc}$ and ${ }^{111}$ In images were reconstructed by selecting photo peak and background energy levels as described before [15].

2.6. Image Quantification. To allow quantification of imaging agent uptake, in vivo isotope-specific conversion factors (CF) were determined for the $0.6 \mathrm{~mm}$ collimator in a representative phantom with a known activity. Using the previously described method [15] the following conversion factors (CF), $\mathrm{CF}_{99 \mathrm{mTc}} 612 \mathrm{MBq} / \mathrm{mL}$ and $\mathrm{CF}_{111 \mathrm{In}} 643 \mathrm{MBq} / \mathrm{mL}$, were found. PMOD 3.7 cardiac tool PCARD (PMOD technologies, Zürich, Switzerland) was used to segment the heart in the 17-segment model. Uptake per segment was subsequently expressed as a mean standardized uptake value $\left(\mathrm{SUV}_{\text {mean }}\right)$, also using the previously described method [15]. For body weight, we assumed that $1 \mathrm{~g}$ equalled $1 \mathrm{~mL}$.

2.7. Biodistribution. After imaging, the vital organs were harvested and kept for gamma-counting (Wallac Wizard, Turku, Finland). Acquired data were expressed as percentage injected dose per gram tissue (\% ID/g).

2.8. CD13 Immunohistological Staining. Hearts were dissected and fixated in HEPES-buffered formaldehyde containing $150 \mathrm{mM}$ saline for $24 \mathrm{~h}$ at $4^{\circ} \mathrm{C}$. Hereafter, hearts 
were placed in $70 \%$ ethanol for maximal one month before embedding in paraffin. Paraffin-embedded hearts were cut at $4 \mu \mathrm{m}$ thickness. Sections were deparaffinized and rehydrated after which endogenous peroxidase was blocked by $0.3 \%$ $\mathrm{H}_{2} \mathrm{O}_{2}$ in methanol. After antigen retrieval (DAKO, target retrieval solution) sections were blocked in 5\% goat serum for one hour. Next, sections were incubated overnight with primary monoclonal antibody against CD13 (1:500, Sigma). After washing, sections were incubated with BrightVision poly-horseradish peroxidase (HRP) Goat anti-Rabbit antibody (Immunologic, Duiven, The Netherlands). HRP was visualized by NOVAred substrate kit (VECTOR Laboratories Inc., Burlingame, CA, USA) and sections were counterstained with hematoxyline. Finally, sections were covered by cover glass with entellan and visualized using Leica Application Suite X (Leica Microsystems, Wetzlar, Germany).

2.9. Statistics. All data were expressed as mean \pm SEM. To test for significant differences we performed an unpaired student's $t$-test with $P<0.05$ being considered statistically significant. Data were analyzed using Microsoft Excel (version 2010).

\section{Results and Discussion}

In this study, an NGR peptide cyclized via native chemical ligation (NCL) and its tetravalent analog were designed and synthesized. The feasibility of these two new NGR peptidebased ligands for radionuclide imaging of CD13 expression in a mouse MI model with SPECT was explored. The peptides were radiolabeled with ${ }^{111}$ In and used for dualisotope SPECT with ${ }^{99 \mathrm{~m}} \mathrm{Tc}$-sestamibi as myocardial perfusion agent. Uptake of ${ }^{99 \mathrm{~m}} \mathrm{Tc}$-sestamibi and ${ }^{111} \mathrm{In}$-coNGR and ${ }^{111}$ In-co(NGR) ${ }_{4}$ was quantified in the 17 -segment model.

3.1. Chemistry. All observed masses of the constructs fell within the range of theoretical monoisotopic and average masses. Structural formulas of final products are given in Figures 2(a)-2(c) and HPLC chromatograms of ${ }^{111}$ In-labeled NGR peptides are displayed in Figure S1. Radiochemical purity generally exceeded $95 \%$ for coNGR, co(NGR) ${ }_{4}$, and cNGR and did not require further purification. From stability studies it appeared that disulfide bond-cyclized cNGR was less stable after 50 minutes of incubation in blood (7.5\% intact product, Table 2) compared to both coNGR-based tracers. The percentage of intact tetrameric coNGR $(25.3 \%)$ is slightly higher compared to coNGR (20.2\%).

Octanol-water partition coefficients indicated that coNGR was more hydrophilic than co(NGR) ${ }_{4}$ (Table 3 ). Remarkably, the reference compound cNGR appeared initially to be the most lipophilic compound $(-3.30 \pm 0.14)$. However, over time its $\log P$ value decreased soon to -4.56 \pm 0.05 (<60 min), most likely explained by instability of this compound. Apparently, backbone cyclization via NCL resulted in a more stable ring structure than cyclization via disulfide bond formation.

3.2. SPECT and Analysis of coNGR and co(NGR) ${ }_{4}$ Uptake. The 17-segment model was used to determine standardized
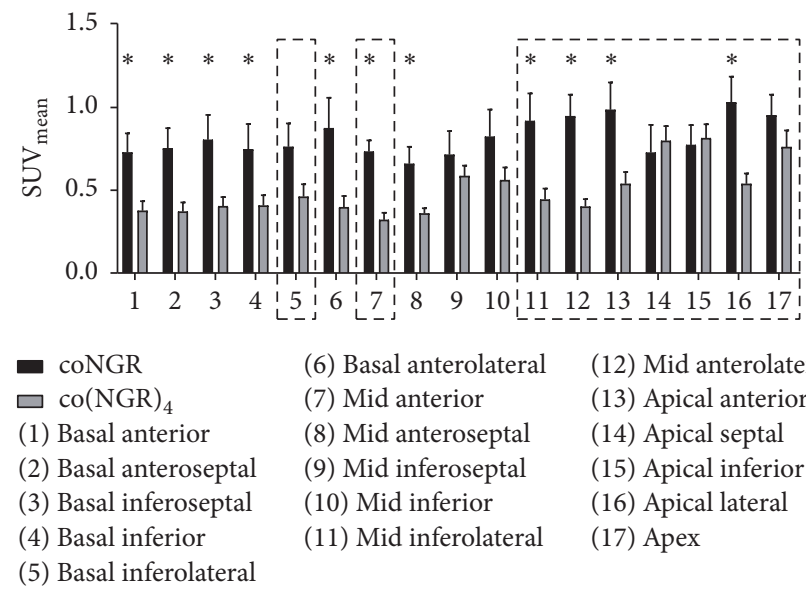

(7) Mid anterior

(8) Mid anteroseptal

(9) Mid inferoseptal

(10) Mid inferior

(11) Mid inferolateral

(12) Mid anterolatera

(13) Apical anterior

(14) Apical septal

(15) Apical inferior

(16) Apical lateral

(17) Apex
(6) Basal anterolateral

FIGURE 3: Overview of the SUVs for coNGR and co(NGR) in MI animals. Infarcted areas (significantly decreased ${ }^{99 \mathrm{~m}}$ Tc-sestamibi uptake) in coNGR group or co(NGR) $)_{4}$ group are encirkeld by the dashed line. ${ }^{*} P<0.05$ is considered statistically significant.

TABLE 2: Blood stability of ${ }^{111}$ In-labeled cyclic NGR-based SPECT tracers coNGR, co(NGR) $)_{4}$, and cNGR [15] after $50 \mathrm{~min}$ of blood incubation.

\begin{tabular}{lc}
\hline Tracer & Intact product (\%) \\
\hline coNGR & 20.22 \\
co(NGR) & 25.31 \\
cNGR & 7.55 \\
\hline
\end{tabular}

TABLE 3: $\log P$ values of ${ }^{111}$ In-labeled coNGR, co(NGR) $)_{4}$, and cNGR [15]. $\log P$ values were determined in three separate experiments, hence indicated as $\log P 1, \log P 2$, and $P 3 . N=3$ for each measurement.

\begin{tabular}{lccc}
\hline Tracer & $\log P_{1}$ & $\log P_{2}$ & $\log P_{3}$ \\
\hline coNGR & $-4.57 \pm 0.06$ & $-4.60 \pm 0.08$ & $-4.54 \pm 0.09$ \\
co(NGR) & $-3.65 \pm 0.02$ & $-3.64 \pm 0.03$ & $-3.84 \pm 0.04$ \\
cNGR & $-3.30 \pm 0.14$ & $-4.17 \pm 0.01$ & $-4.56 \pm 0.05$ \\
\hline
\end{tabular}

uptake values (SUVs) of the tracers [24]. ${ }^{99} \mathrm{~m}$ Tc-sestamibi allowed visualization of infarcted myocardium 7 days after LAD ligation. Infarcted areas were defined as areas with significantly reduced uptake of ${ }^{99 \mathrm{~m}} \mathrm{Tc}$-sestamibi in either coNGR or $\mathrm{co}(\mathrm{NGR})_{4}$ injected mice with LAD ligation compared to sham-operated mice (Table S2). Highest uptake of ${ }^{111}$ In-coNGR and ${ }^{111}$ In-co(NGR) $)_{4}$ was observed in the area in and around the apex, which corresponds to the infarct area (Figures 3 and 4 and Table S3). Uptake patterns of coNGR and co(NGR $)_{4}$ can be seen in Figures 5 and 6, respectively.

To examine whether multimerization indeed resulted in higher target uptake, SUVs of co(NGR) ${ }_{4}$ and coNGR in infarcted and noninfarcted areas were compared for each tracer separately. Uptake of co(NGR $)_{4}$ in segments that were affected by MI was significantly higher $(0.57 \pm 0.03)$ than in segments that were not affected by MI $(0.43 \pm 0.02)$, whereas uptake of coNGR in MI-affected segments $(0.83 \pm 0.05)$ was not significantly higher than in unaffected segments $(0.76 \pm$ 


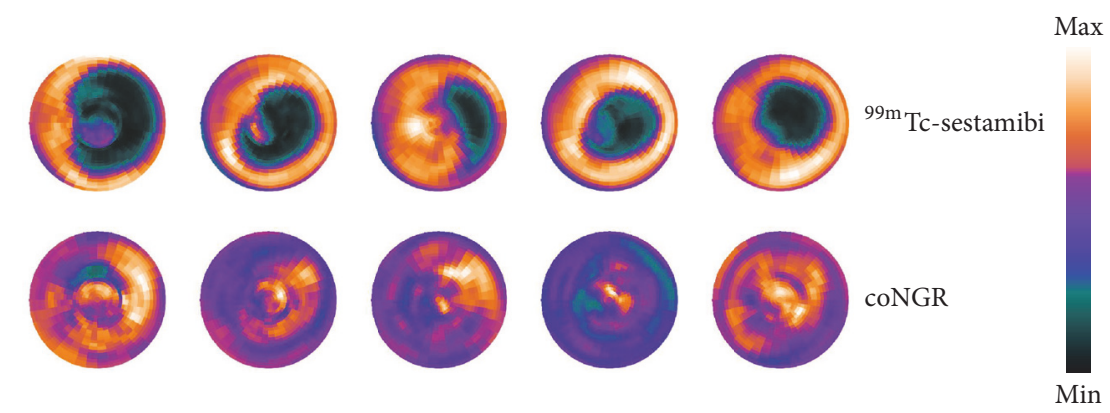

(a)
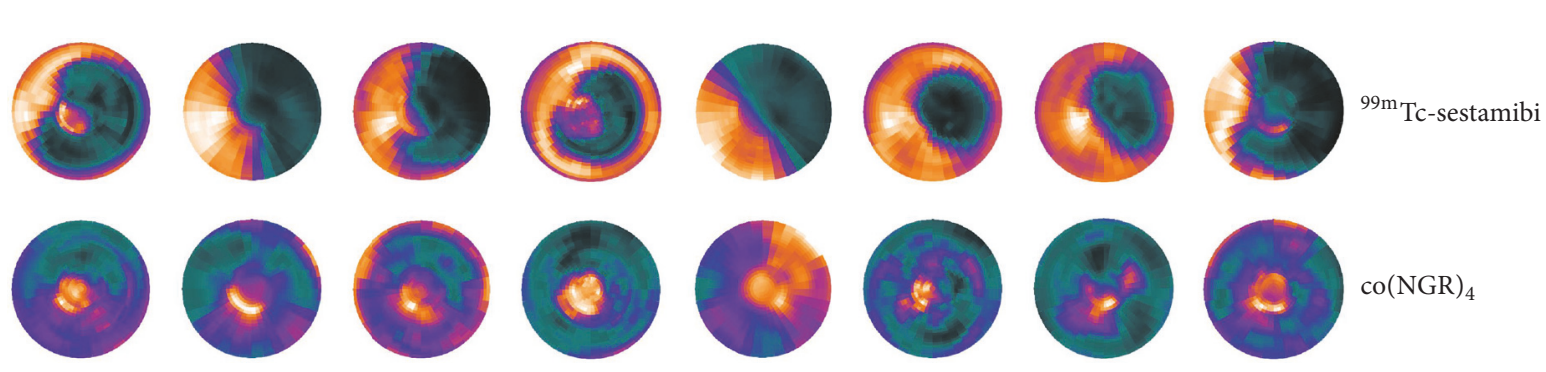

(b)

FIGURE 4: Polar perfusion maps from each mouse in the MI group displaying the uptake pattern of ${ }^{99 \mathrm{~m}_{\mathrm{m}}}$ Tc-sestamibi and coNGR (a) or ${ }^{99 \mathrm{~m}}$ Tcsestamibi and co(NGR) $)_{4}$ (b). The uptake in the polar perfusion maps is color coded and relative to the injected dose per animal.

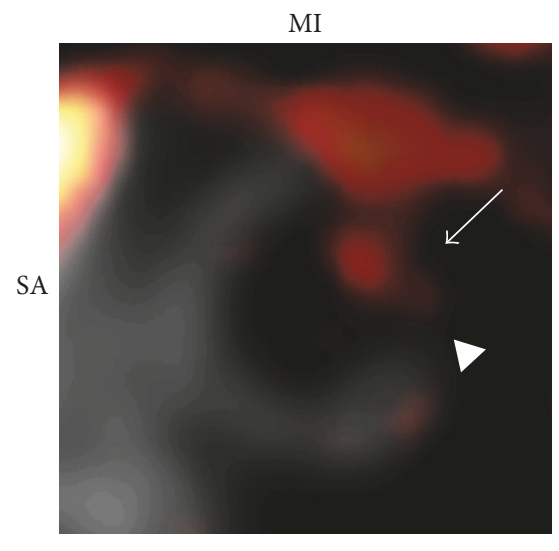

(a)

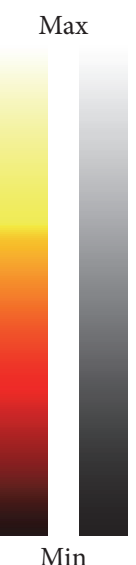

Min

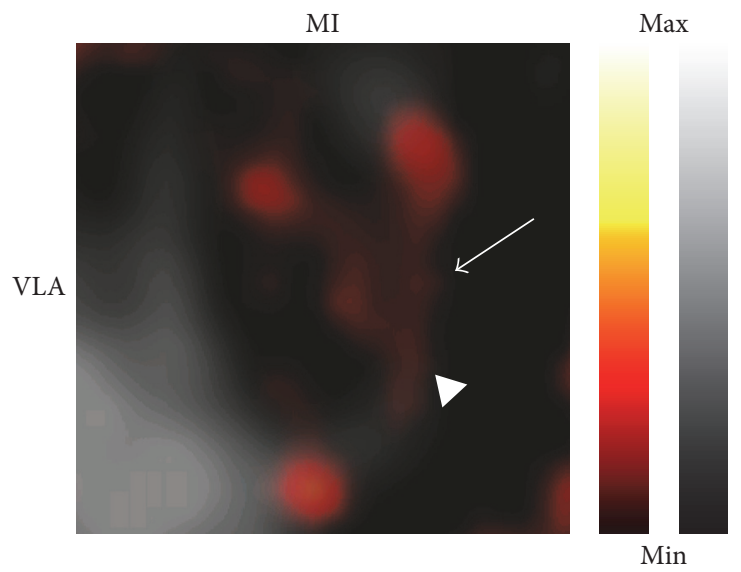

(b)

FIGURE 5: Representative in vivo fusion images of ${ }^{99 \mathrm{~m}}$ Tc-sestamibi and coNGR uptake in MI mice. In "grey" is the uptake of ${ }^{99 \mathrm{~m}}$ Tc-sestamibi in myocardium, while uptake of coNGR is in "hot metal" color. In (a) and (b) slices of an infarcted heart are shown. The infarct area is visualized by the decreased uptake of ${ }^{99 \mathrm{~m}} \mathrm{Tc}$-sestamibi (in grey) which is clearly visible in the anterolateral region of the heart (arrowheads). Enhanced uptake of coNGR is clearly visible in the infarct area (arrows). Note: the uptake is color coded and relative to the injected dose per animal. SA: short axis; VLA: vertical long axis.

0.05). This suggests that $\mathrm{co}(\mathrm{NGR})_{4}$ has a more specific uptake in infarcted areas than coNGR.

Although coNGR and co(NGR) $)_{4}$ were not directly compared with cNGR in an in vivo study, both coNGR-based ligands showed a higher uptake than cNGR in the infarcted area. However, only $\mathrm{co}(\mathrm{NGR})_{4}$ indicated a more targetspecific uptake which makes $\mathrm{co}(\mathrm{NGR})_{4}$ a more optimal agent than cNGR or coNGR for imaging of angiogenesis after MI.
3.3. Biodistribution of coNGR and $\mathrm{co}(\mathrm{NGR})_{4}$. One hour postinjection (p.i.), urinary excretion in MI mice was $72.5 \pm$ 5.2 for coNGR and $54.9 \pm 9.1$ for $\mathrm{co}(\mathrm{NGR})_{4}(\% \mathrm{ID} / \mathrm{g} \pm \mathrm{SEM}$, $P=0.23)$. Retention of coNGR appeared to be significantly higher in blood, muscle, lungs, and intestines compared to retention of $\mathrm{co}(\mathrm{NGR})_{4}$. It could be that $\mathrm{co}(\mathrm{NGR})_{4}$ clears a bit more via the hepotobiliary route than coNGR, though liver uptake of $\mathrm{co}(\mathrm{NGR})_{4}$ and coNGR was not statiscally different. 


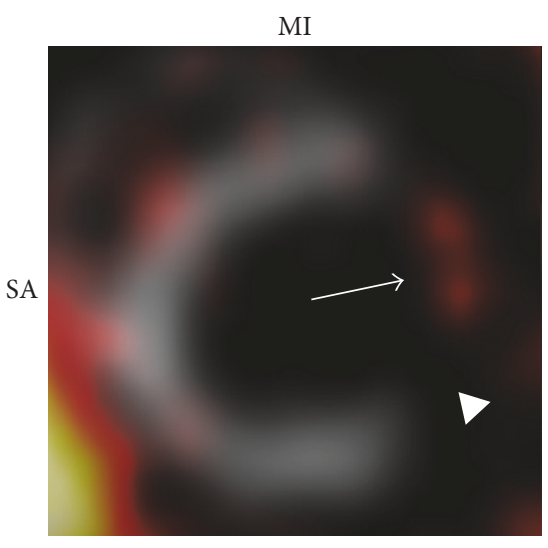

(a)

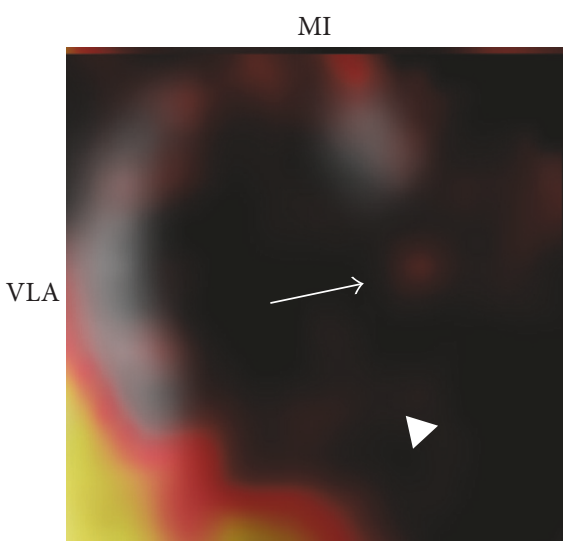

(c)
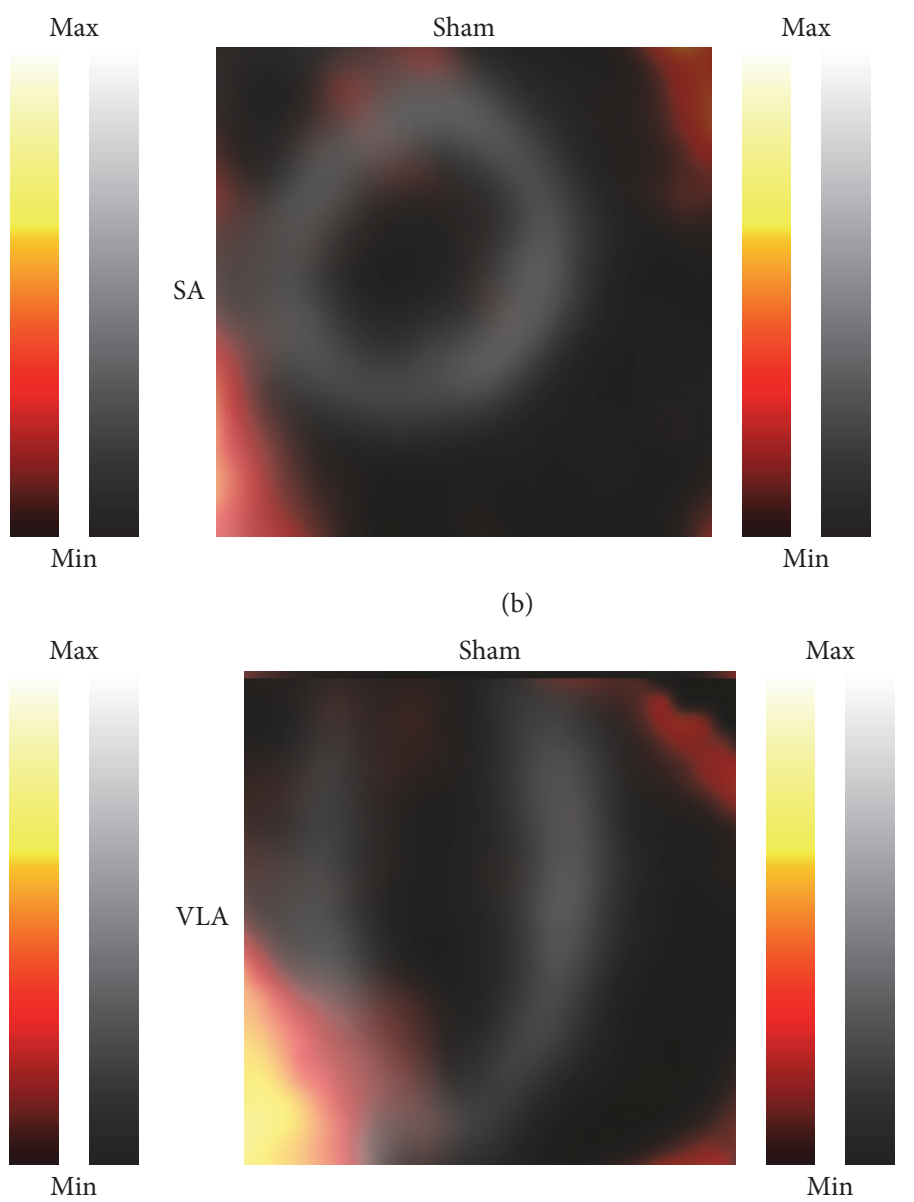

(d)

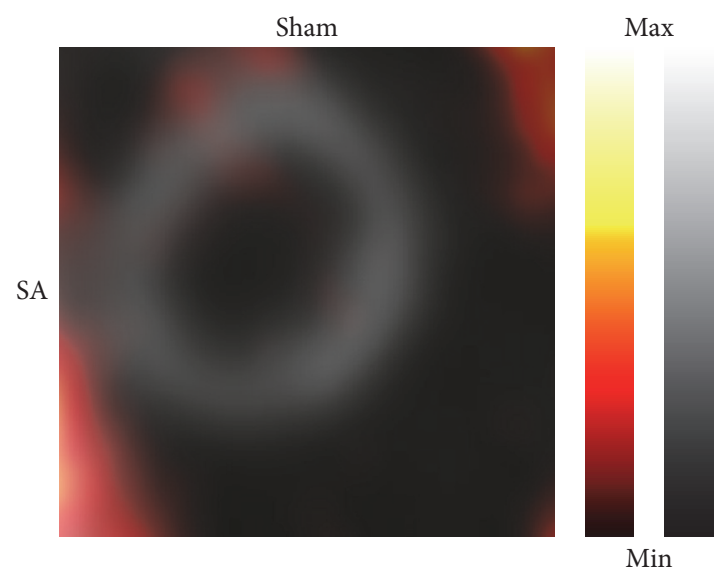

(b)

FIGURE 6: Representative in vivo fusion images of ${ }^{99 \mathrm{~m}}$ Tc-sestamibi and co(NGR) ${ }_{4}$ uptake. In "grey" is the uptake of ${ }^{99 \mathrm{~m}}$ Tc-sestamibi in myocardium, while uptake of co(NGR) ${ }_{4}$ imaging is in "hot metal" color. (a) and (c): infarcted heart. Infarct area is signified by decreased uptake of ${ }^{99 \mathrm{~m}} \mathrm{Tc}$-sestamibi which is clearly visible in the anterolateral region of the heart (arrowheads). Enhanced uptake of co(NGR) ${ }_{4}$ is clearly visibly in the infarct area (arrows). (b) and (d): sham-operated heart. Uniform uptake of ${ }^{99 \mathrm{~m}} \mathrm{Tc}$-sestamibi can be seen in combination with low overall uptake of co(NGR $)_{4}$. Note: uptake is color coded and relative to injected dose per animal. SA: short axis; VLA: vertical long axis.

Other organs displayed a low uptake of coNGR that did not

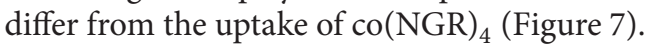

Unlike with cyclic RGD, cyclic NGR multimerization did not result in higher target uptake as coNGR exceeded $\mathrm{co}(\mathrm{NGR})_{4}$ uptake. Changing the currently used short SMCC spacer, which only allows statistical rebinding, for a longer and less rigid spacer that might enable binding to multiple CD13 receptors simultaneously, could improve the avidity and thereby the affinity of the tracer. For example, a flexible PEG spacer with a length that could bridge the width of the CD13 receptor of $131 \AA[25]$ might be suitable.

3.4. CD13 Immunohistochemistry. To validate uptake patterns of both coNGR-based tracers, cardiac CD13 expression was evaluated through immunohistological staining. A low level of CD13 expression was observed within the myocardium of sham-operated control animals (Figures $8(a)$ and $8(d)$ ). 


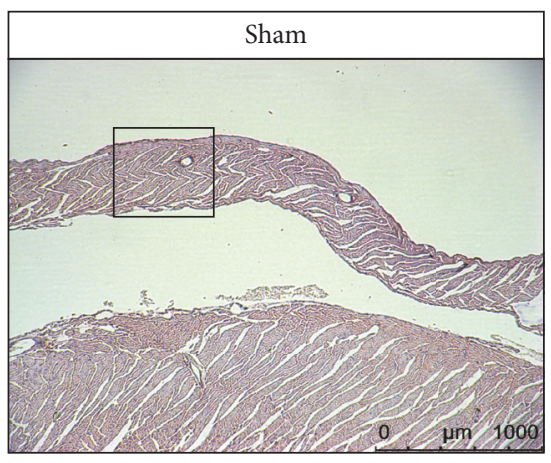

(a)

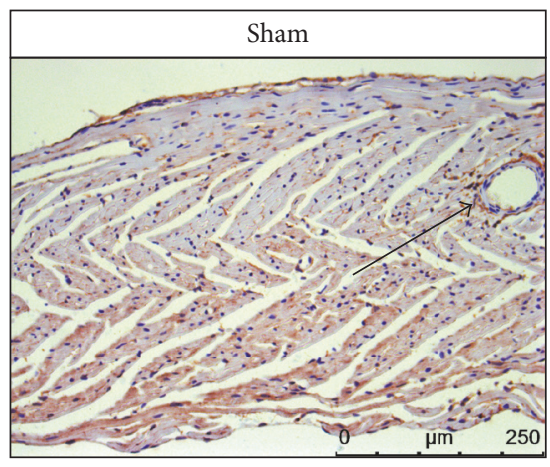

(d)

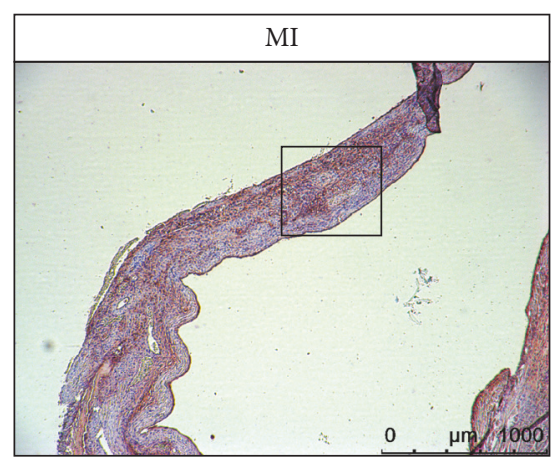

(b)

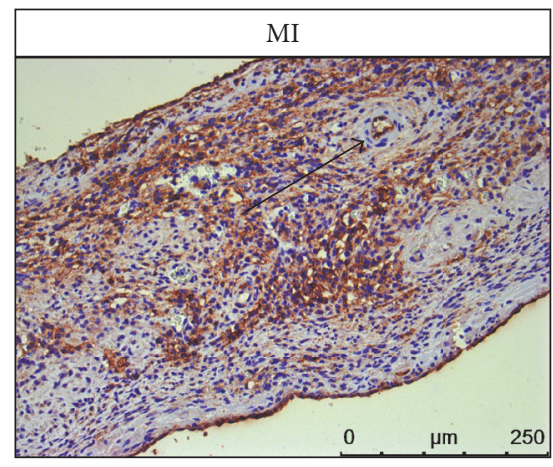

(e)

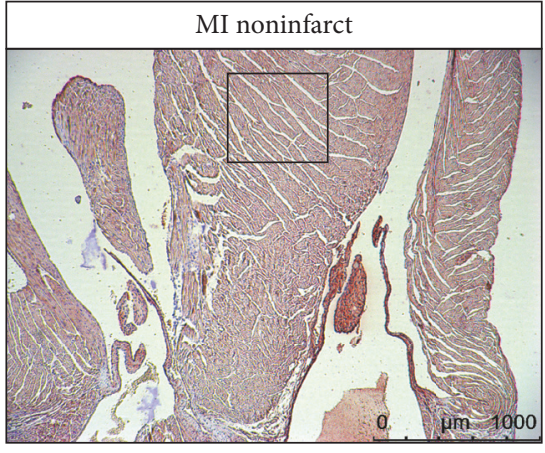

(c)

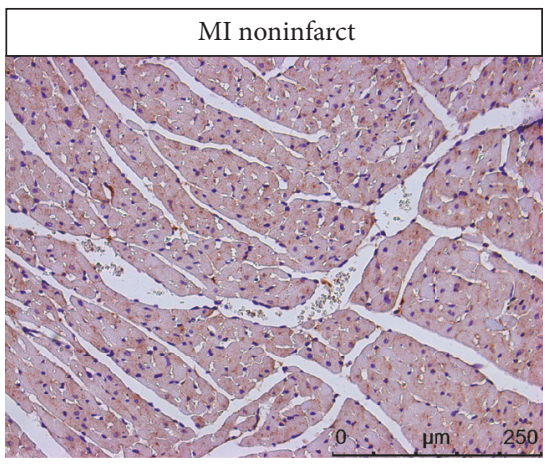

(f)

FIgURE 8: Representative pictures of CD13 expression in myocardium of sham and MI operated animals. Low baseline expression of CD13 was observed on photomicrographs (magnification 40x) of the myocardium of sham-operated animals (a). Dramatically increased CD13 expression was observed on photomicrographs (magnification 40x) of infarcted myocardium of MI operated animals (b). On photomicrographs (magnification 40x) of noninfarcted myocardium, a marginally higher expression of CD13 was noticed (c). Photomicrographs (magnification 200x) in (d), (e), and (f) represent a magnification of the region indicated in (a), (b), and (c), respectively. Note that expression of CD13 is not restricted to blood vessels but that endothelium of blood vessels is positive in the infarcted heart as opposed to the sham (arrows).

While the level of CD13 expression in the noninfarcted myocardium of MI animals appeared marginally higher compared to the expression level in control animals (Figures $8(\mathrm{c})$ and $8(\mathrm{f}))$, the level of CD13 expression was markedly higher in the infarcted myocardium of MI animals (Figures $8(\mathrm{~b})$ and $8(\mathrm{e}))$.

The enhanced uptake of coNGR and co(NGR) $)_{4}$ in the healthy myocardium of infarcted hearts points towards an overall angiogenic response of the heart in MI animals. Additionally, for co(NGR) $)_{4}$ the highest level of uptake was found in and around the infarcted areas of MI animals which also correlated to the histological findings as the highest level of CD13 expression was found in and around the infarcted areas. The same trend was observed for coNGR. It is highly likely that the uptake in the infarcted area as well as in the healthy myocardium is specific and related to increased CD13 expression.

\section{Conclusions}

Two CD13-targeting SPECT tracers for angiogenesis, coNGR and $\mathrm{co}(\mathrm{NGR})_{4}$, were designed and synthesized. However, target uptake of cyclic NGR-based imaging agents does not seem to increase with increasing valency. Instead, the key to enhance cyclic NGR-based imaging agent uptake was to stabilize the ring structure through NCL. Additional studies with different linkers conjugated to the lysine scaffold are warranted to investigate a possible increased avidity effect with coNGR-based imaging agents.

\section{Conflicts of Interest}

The authors declare that there are no conflicts of interest regarding the publication of this article.

\section{Acknowledgments}

This study was performed within the framework of the Center for Translational Molecular Medicine (CTMM), Project EMINENCE (Grant 01C-204 to Geert Hendrikx, Tilman M. Hackeng, Mark J. Post, and Ingrid Dijkgraaf), the Weijerhorst Foundation (Matthias Bauwens; Felix M. Mottaghy), and The Netherlands Organisation for Scientific Research (NWO; VIDI 723.013.009 to Ingrid Dijkgraaf). CTMM, Weijerhorst Foundation, and NWO are cordially acknowledged for their financial support. 


\section{References}

[1] P. Carmeliet, "Angiogenesis in life, disease and medicine," Nature, vol. 438, no. 7070, pp. 932-936, 2005.

[2] F. A. Jaffer, D. E. Sosnovik, M. Nahrendorf, and R. Weissleder, "Molecular imaging of myocardial infarction," Journal of Molecular and Cellular Cardiology, vol. 41, no. 6, pp. 921-933, 2006.

[3] A. A. Kocher, M. D. Schuster, M. J. Szabolcs et al., "Neovascularization of ischemic myocardium by human bone-marrowderived angioblasts prevents cardiomyocyte apoptosis, reduces remodeling and improves cardiac function," Nature Medicine, vol. 7, no. 4, pp. 430-436, 2001.

[4] R. C. Hendel, T. D. Henry, K. Rocha-Singh et al., "Effect of intracoronary recombinant human vascular endothelial growth factor on myocardial perfusion: Evidence for a dose-dependent effect," Circulation, vol. 101, no. 2, pp. 118-121, 2000.

[5] T. D. Henry, B. H. Annex, G. R. McKendall et al., "The VIVA trial: vascular endothelial growth factor in ischemia for vascular angiogenesis," Circulation, vol. 107, no. 10, pp. 1359-1365, 2003.

[6] T. D. Henry, K. Rocha-Singh, J. M. Isner et al., "Intracoronary administration of recombinant human vascular endothelial growth factor to patients with coronary artery disease," American Heart Journal, vol. 142, no. 5, pp. 872-880, 2001.

[7] M. Simons, B. H. Annex, R. J. Laham et al., "Pharmacological treatment of coronary artery disease with recombinant fibroblast growth factor-2: Double-blind, randomized, controlled clinical trial," Circulation, vol. 105, no. 7, pp. 788-793, 2002.

[8] M. Simons, "Angiogenesis: Where do we stand now?" Circulation, vol. 111, no. 12, pp. 1556-1566, 2005.

[9] M. R. Stacy, M. W. Maxfield, and A. J. Sinusas, "Targeted molecular imaging of angiogenesis in PET and SPECT: a review," Yale Journal of Biology and Medicine, vol. 85, no. 1, pp. 75-86, 2012.

[10] M. R. Stacy, J. C. Paeng, and A. J. Sinusas, “The role of molecular imaging in the evaluation of myocardial and peripheral angiogenesis," Annals of Nuclear Medicine, vol. 29, no. 3, pp. 217-223, 2015.

[11] G. Hendrikx, S. Vöö, M. Bauwens, M. J. Post, and F. M. Mottaghy, "SPECT and PET imaging of angiogenesis and arteriogenesis in pre-clinical models of myocardial ischemia and peripheral vascular disease," European Journal of Nuclear Medicine and Molecular Imaging, vol. 43, no. 13, pp. 2433-2447, 2016.

[12] W. Arap, R. Pasqualini, and E. Ruoslahti, "Cancer treatment by targeted drug delivery to tumor vasculature in a mouse model," Science, vol. 279, no. 5349, pp. 377-380, 1998.

[13] A. Buehler, M. A. M. J. Van Zandvoort, B. J. Stelt et al., "CNGR: a novel homing sequence for CD13/APN targeted molecular imaging of murine cardiac angiogenesis in vivo," Arteriosclerosis, Thrombosis, and Vascular Biology, vol. 26, no. 12, pp. 2681-2687, 2006.

[14] M. Oostendorp, K. Douma, A. Wagenaar et al., "Molecular magnetic resonance imaging of myocardial angiogenesis after acute myocardial infarction," Circulation, vol. 121, no. 6, pp. 775783, 2010.

[15] G. Hendrikx, M. De Saint-Hubert, I. Dijkgraaf et al., "Molecular imaging of angiogenesis after myocardial infarction by 111InDTPA-cNGR and 99mTc-sestamibi dual-isotope myocardial SPECT," EJNMMI Research, vol. 5, no. 1, 2015.

[16] I. Dijkgraaf, A. Y. Rijnders, A. Soede et al., "Synthesis of DOTA-conjugated multivalent cyclic-RGD peptide dendrimers via 1,3-dipolar cycloaddition and their biological evaluation: implications for tumor targeting and tumor imaging purposes," Organic \& Biomolecular Chemistry, vol. 5, no. 6, pp. 935-944, 2007.

[17] Z.-B. Li, K. Chen, and X. Chen, "68Ga-labeled multimeric RGD peptides for microPET imaging of integrin $\alpha \mathrm{v} \beta 3$ expression," European Journal of Nuclear Medicine and Molecular Imaging, vol. 35, no. 6, pp. 1100-1108, 2008.

[18] T. M. Hackeng, J. H. Griffin, and P. E. Dawson, "Protein synthesis by native chemical ligation: expanded scope by using straightforward methodology," Proceedings of the National Acadamy of Sciences of the United States of America, vol. 96, no. 18, pp. 10068-10073, 1999.

[19] M. Schnölzer, P. Alewood, A. Jones, D. Alewood, and S. B. H. Kent, "In situ neutralization in Boc-chemistry solid phase peptide synthesis: rapid, high yield assembly of difficult sequences," International Journal of Peptide and Protein Research, vol. 40, no. 3-4, pp. 180-193, 1992.

[20] A. Dirksen, E. W. Meijer, W. Adriaens, and T. M. Hackeng, "Strategy for the synthesis of multivalent peptide-based nonsymmetric dendrimers by native chemical ligation," Chemical Communications, no. 15, pp. 1667-1669, 2006.

[21] P. Van de Vijver, D. Suylen, A. Dirksen, P. E. Dawson, and T. M. Hackeng, "Nepsilon-(thiaprolyl)-lysine as a handle for sitespecific protein conjugation.," Biopolymers, vol. 94, no. 4, pp. 465-474, 2010.

[22] I. Dijkgraaf, G. M. Franssen, W. J. McBride et al., "PET of tumors expressing gastrin-releasing peptide receptor with an 18F-labeled bombesin analog," Journal of Nuclear Medicine, vol. 53, no. 6, pp. 947-952, 2012.

[23] E. Lutgens, M. J. A. P. Daemen, E. D. De Muinck, J. Debets, P. Leenders, and J. F. M. Smits, "Chronic myocardial infarction in the mouse: cardiac structural and functional changes," Cardiovascular Research, vol. 41, no. 3, pp. 586-593, 1999.

[24] M. D. Cerqueira, N. J. Weissman, V. Dilsizian et al., "Standardized myocardial segmentation and nomenclature for tomographic imaging of the heart," Circulation, vol. 105, pp. 539-542, 2001.

[25] A. H. M. Wong, D. Zhou, and J. M. Rini, “The x-ray crystal structure of human aminopeptidase $\mathrm{N}$ reveals a novel dimer and the basis for peptide processing," The Journal of Biological Chemistry, vol. 287, no. 44, pp. 36804-36813, 2012. 


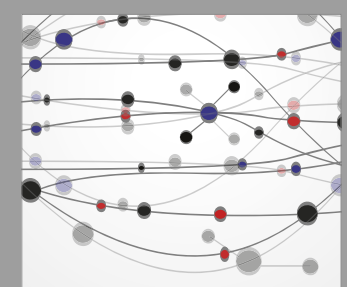

The Scientific World Journal
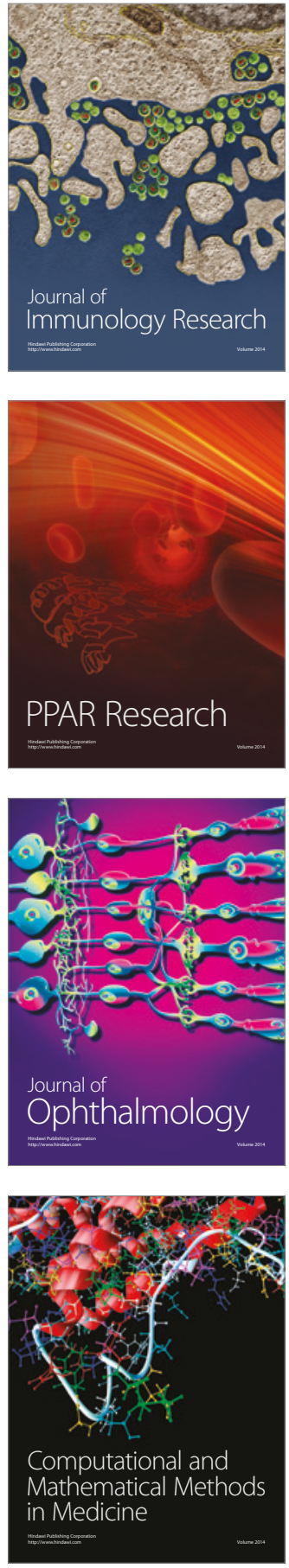

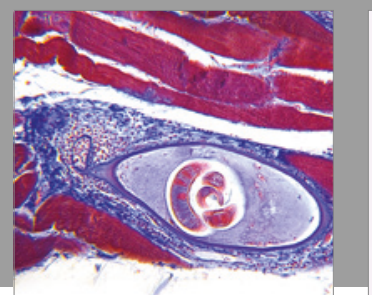

Gastroenterology Research and Practice
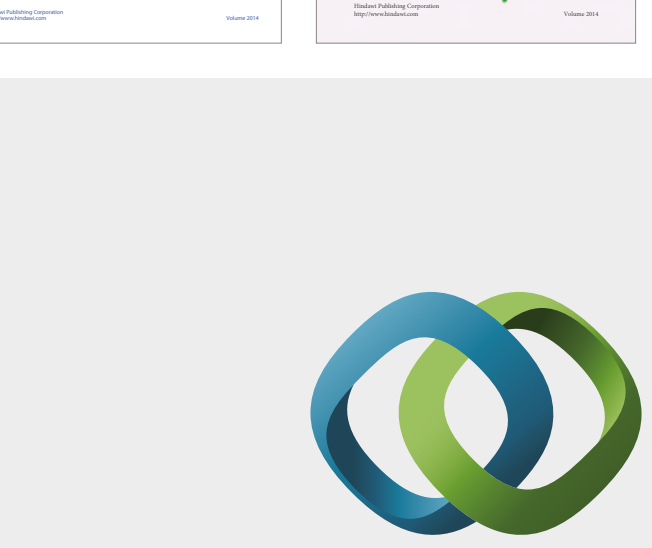

\section{Hindawi}

Submit your manuscripts at

https://www.hindawi.com
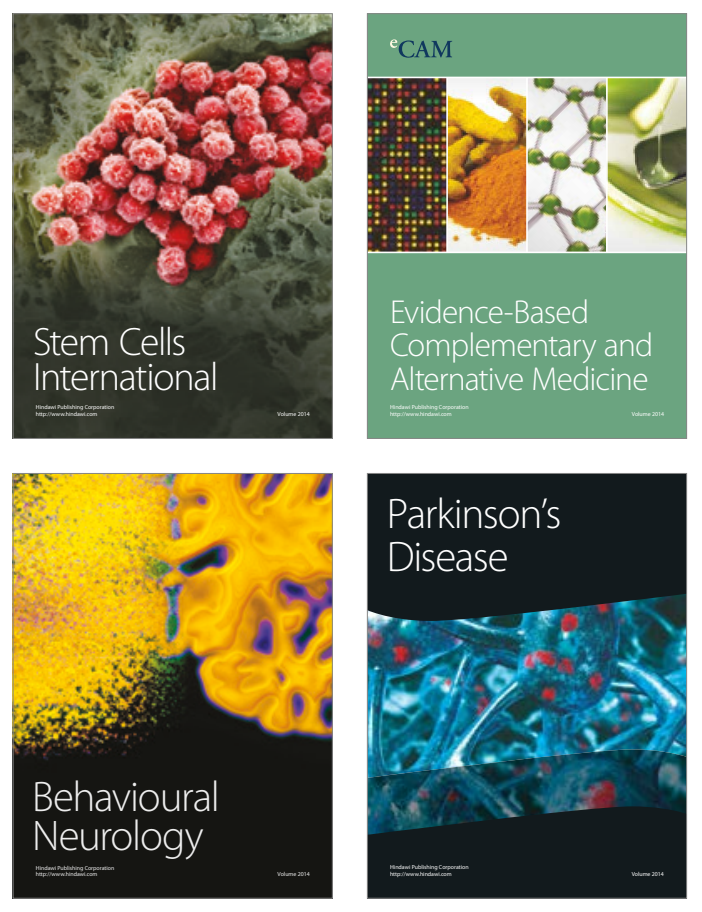
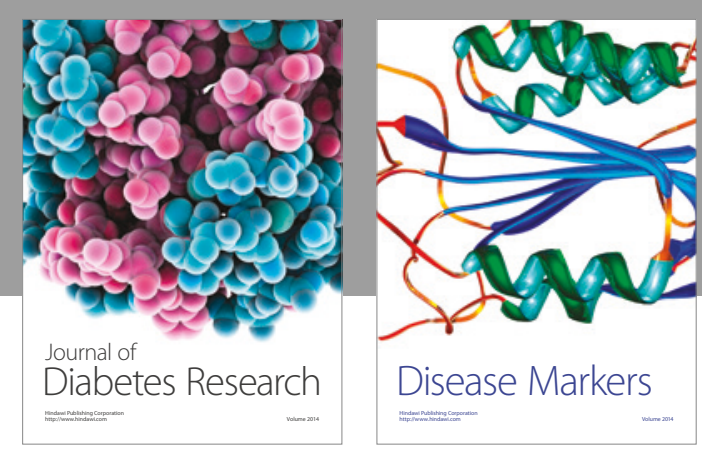

Disease Markers
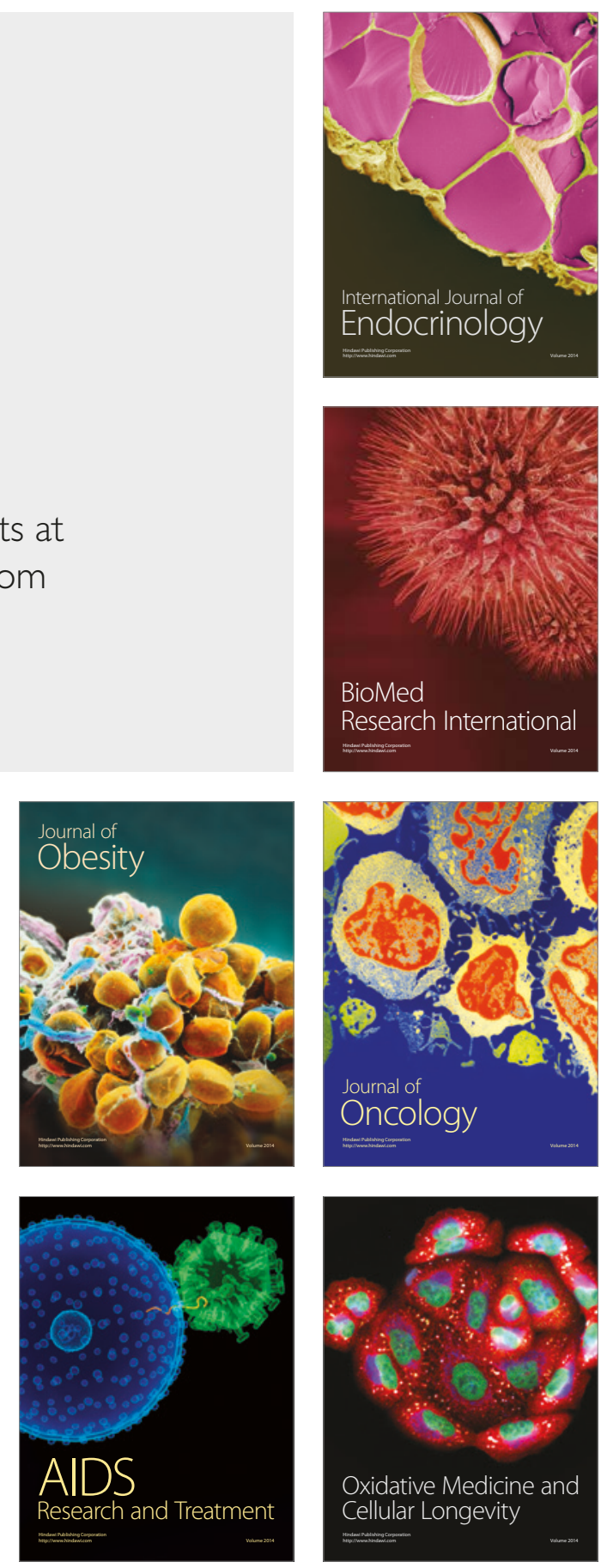